\title{
Epigenetic regulation of placental gene expression in transcriptional subtypes of preeclampsia
}

\author{
Katherine Leavey', Samantha L. Wilson ${ }^{2,3}$, Shannon A. Bainbridge ${ }^{4,5}$, Wendy P. Robinson ${ }^{2,3}$ and Brian J. Cox ${ }^{1,6^{*}}$
}

\begin{abstract}
Background: Preeclampsia (PE) is a heterogeneous, hypertensive disorder of pregnancy, with no robust biomarkers or effective treatments. We hypothesized that this heterogeneity is due to the existence of multiple subtypes of PE and, in support of this hypothesis, we recently identified five clusters of placentas within a large gene expression microarray dataset $(N=330)$, of which four (clusters $1,2,3$, and 5$)$ contained a substantial number of PE samples. However, while transcriptional analysis of placentas can subtype patients, we propose that the addition of epigenetic information could discern gene regulatory mechanisms behind the distinct PE pathologies, as well as identify clinically useful potential biomarkers.
\end{abstract}

Results: We subjected 48 of our samples from transcriptional clusters 1, 2, 3, and 5 to Infinium HumanMethylation450 arrays. Samples belonging to transcriptional clusters 1-3 still showed visible relationships to each other by methylation, but cluster 5 , with known chromosomal abnormalities, no longer formed a cohesive group. Within transcriptional clusters 2 and 3, controlling for fetal sex and gestational age in the identification of differentially methylated sites, compared to the healthier cluster 1, dramatically reduced the number of significant sites, but increased the percentage that demonstrated a strong linear correlation with gene expression (from 5\% and 2\% to 9\% and 8\%, respectively). Locations exhibiting a positive relationship between methylation and gene expression were most frequently found in $\mathrm{CpG}$ open sea enhancer regions within the gene body, while those with a significant negative correlation were often annotated to the promoter in a $\mathrm{CpG}$ shore region. Integrated transcriptome and epigenome analysis revealed modifications in TGF-beta signaling, cell adhesion, oxidative phosphorylation, and metabolism pathways in cluster 2 placentas, and aberrations in antigen presentation, allograft rejection, and cytokine-cytokine receptor interaction in cluster 3 samples.

Conclusions: Overall, we have established DNA methylation alterations underlying a portion of the transcriptional development of "canonical" PE in cluster 2 and "immunological" PE in cluster 3. However, a significant number of the observed methylation changes were not associated with corresponding changes in gene expression, and vice versa, indicating that alternate methods of gene regulation will need to be explored to fully comprehend these PE subtypes.

Keywords: Preeclampsia, Placenta, DNA methylation, Gene expression, Clustering, Subtypes

\section{Background}

Preeclampsia $(\mathrm{PE})$ is a complex, heterogeneous disorder of pregnancy, diagnosed by the onset of maternal hypertension after the 20th week of gestation, with signs of maternal multi-organ dysfunction [1]. As with many

\footnotetext{
* Correspondence: b.cox@utoronto.ca

'Department of Physiology, University of Toronto, 1 King's College Circle, Toronto, ON, Canada

${ }^{6}$ Department of Obstetrics and Gynecology, University of Toronto, 23 Edward Street, Toronto, ON, Canada

Full list of author information is available at the end of the article
}

pathologies of pregnancy, PE has no cure, robust predictive biomarkers, or effective treatments, other than the delivery of the infant to discontinue the pregnancy and remove what is thought to be the causative organ, the placenta. Repeated attempts to characterize the placental molecular pathology and identify biomarkers of PE by applying a binary approach (PE versus control) have not been clinically fruitful, and we hypothesized that this is due to the existence of multiple molecular subtypes of PE [2].

In support of this hypothesis, we recently published a large unsupervised clustering analysis of microarray data 
from a PE-focused placental cohort $(N=330)$, including 157 highly annotated samples purchased from a single biobank [3]. This revealed five clusters of placental gene expression containing at least three clinically significant etiological subtypes of PE: "maternal", with term and near-term deliveries of average-sized infants and placentas that appear molecularly similar to normal healthy control samples; "canonical" with high placental expression of known PE markers, preterm deliveries, low fetal weights, and evidence of maternal malperfusion; and "immunological" with severe fetal growth restriction, enrichment of immune response genes, and histological signs of maternal anti-fetal/placental rejection [3], belonging to transcriptional clusters 1, 2, and 3, respectively. An additional subtype of PE placentas with chromosomal abnormalities was also discovered within cluster 5 (and supported by array-based comparative genomic hybridization (aCGH) analysis), but showed no strong clinical association [3].

However, despite our considerable progress towards understanding the molecular diversity observed amongst PE patients, RNA is relatively unstable, easily affected by technical variability [4], and rarely successful as a therapeutic target [5], limiting its clinical utility. We, therefore, propose that the integration of an additional level of molecular information in these placentas, such as DNA methylation, will compensate for these restrictions [4], as well as improve our understanding of the molecular pathology.

DNA methylation is a mitotically heritable epigenetic mark employed by the cell to control gene expression without altering the genetic sequence [6], although the relationship between the two data types is exceptionally complex [7-10]. Given the flexibility for modification in the epigenome, these methylation events may also serve to provide insight into the environmental exposures sustained by the cell [11], and as potential biomarkers of early cellular transformations [12]. In fact, many examples exist, particularly in the cancer field, for the exploitation of DNA methylation in the diagnosis, prognosis, and prediction of drug response in disease [12, 13], and as possible therapeutic targets [14, 15].

Here, we subject a subset of our highly annotated cohort samples to DNA methylation arrays and investigate differences in the placental methylome between our previously identified transcriptional clusters, as well as relationships between the two data types. Furthermore, by assessing epigenetic changes associated with the observed pathological gene expression, we also attempt to discover novel therapeutic targets for the various PE subtypes.

\section{Methods}

\section{Sample selection}

A total of 48 (out of 157) placentas from our highly annotated cohort purchased from the Research Centre for
Women's and Infants' Health (RCWIH) BioBank [3] were selected for DNA methylation analysis (19 from transcriptional cluster 1, 19 from transcriptional cluster 2, 5 from transcriptional cluster 3 , and 5 from transcriptional cluster 5), using the sample function in $\mathrm{R} 3.1 .3$ (Additional file 1: Figure S1). The selected number of samples per cluster is approximately representative of the sample distribution in the full placental dataset, with the condition of a minimum of five samples per cluster. Our cohort selection and tissue sampling methods have been previously described [3]. Placentas demonstrating signs of chorioamnionitis or belonging to the chorioamnionitis-associated transcriptional cluster 4 [3] were not included as these are a known entity, independent of preeclampsia (Additional file 1: Figure S1). Clinical differences between these 48 patients only were assessed using Kruskal-Wallis rank sum, Wilcoxon rank sum, and Fisher's exact tests, as appropriate.

\section{Methylation arrays and data processing}

DNA was isolated from the 48 placentas by ethanol precipitation with the Wizard ${ }^{\circledR}$ Genomic DNA Purification Kit from Promega and quantified by a NanoDrop 1000 spectrophotometer. A total of 750 ng of DNA per sample was bisulfite converted using the EZ Gold DNA methylation kit (Zymo) and assessed for methylation status with Infinium HumanMethylation450 arrays from Illumina. This array covers $\mathrm{CpG}$ islands (tight clusters of CpG sites) as well as shores (up to $2 \mathrm{~kb}$ from $\mathrm{CpG}$ islands), shelves (2-4 kb from CpG islands) and open sea ( $>4 \mathrm{~kb}$ from CpG islands) [16]. Arrays were scanned by an Illumina HiScan 2000. This methylation data was also used as a validation cohort in [17].

The resulting IDAT files were loaded into $\mathrm{R}$ using the champ.load function (ChAMP library) [18], excluding low quality probes with a detection $p$ value above 0.01 in more than one sample or a beadcount $<3$ in at least $5 \%$ of samples $(N=1940)$. Probes known to bind sex chromosomes, cross-hybridize to multiple locations, or target a single-nucleotide polymorphism (SNP) were removed, based on previous annotation [19, 20]. This left 410,664 probes for DNA methylation analysis. The samples underwent functional normalization with the preprocessFunnorm function [21], which is an extension of quantile normalization utilizing the control probes on the array, applied separately to the methylated and unmethylated intensities, type I and type II signals, and the male and female samples. The data was then batch corrected for slide and array position using the ComBat function (sva library) [22] without accounting for any outcome of interest or other covariates to obtain the most unbiased results. All analysis was performed using $M$ values to improve the statistical calculation of 
differential methylation [23, 24], although beta values are also included in the tables for biological interpretation.

\section{Gene expression data processing}

Our entire 157 placenta dataset was previously hybridized against Human Gene 1.0 ST Array chips from Affymetrix [3]. The resulting microarray CEL files for the 48 placentas assessed for methylation in the current study were loaded into $\mathrm{R}$, and normalized and converted to $\log 2$ values using the affy library [25]. Expression values annotated to the same gene symbol were merged to a mean value, and genes with expression in the lowest quartile were filtered out to reduce confounding by background noise, using the varFilter function.

\section{Identification of differentially methylated positions}

The global relationships between the 48 samples based on the DNA methylation information alone were visualized using $\mathrm{t}$-distributed stochastic neighbor embedding ( $\mathrm{t}-\mathrm{SNE}$; tsne library) [26] with a perplexity of 10 . Samples belonging to our previously described transcriptional clusters 2,3 , and 5 were compared to cluster 1 placentas (with a "healthy" transcriptional profile) to identify differentially methylated positions, using the limma library [27]. The entire cluster 1 was employed as the comparison group after confirming that no significant differentially methylated positions exist between the PE and normotensive controls within cluster 1 by limma, and no segregation of these phenotype groups within cluster 1 were observed by t-SNE (Fig. 1). Linear modeling, compared to cluster 1, was performed both with and without controlling for fetal sex (male or female) and/or gestational age (GA) at delivery
(26-40 weeks) to investigate the impact of these variables on each cluster. Fetal sex was still considered despite the removal of the sex chromosomes from the analysis due to likely persistent differences on the autosomes [28, 29]. Sites were considered differentially methylated at a false discovery rate (FDR) corrected $q$ value $<0.05$, and groups of significant positions were noted when at least three significant sites were identified within 1000 base pairs of each other.

\section{Probe annotation and epigenetic regulation of gene expression}

All DNA methylation probes were assigned to enhancer regions, CpG regions (island, shore, shelf, or open sea), and gene-centric locations (TSS1500: 200-1500 nucleotides upstream of the transcriptional start site (TSS); TSS200: TSS to 200 nucleotides upstream of the TSS; 5 ' untranslated region (UTR); 1st exon; gene body; 3'UTR; and intergenic region (IGR)) based on the IlluminaHumanMethylation450kanno.ilmn12.hg19 library. A number of sites $(N=45,354)$ were linked to multiple genes or gene aliases, and all possible associations were maintained. Probes found in the IGR were assigned to the gene with the closest TSS. Trends in significant probe positions were assessed by Fisher's exact tests.

Sites identified as significantly differentially methylated in transcriptional cluster 2 or 3 placentas, compared to cluster 1 samples, were investigated for linear correlations between the $M$ values and the corresponding log2 gene expression values within the relevant two clusters. Correlations were considered significant at a FDR $<0.05$ and correlation groups were compared by Fisher's exact tests.
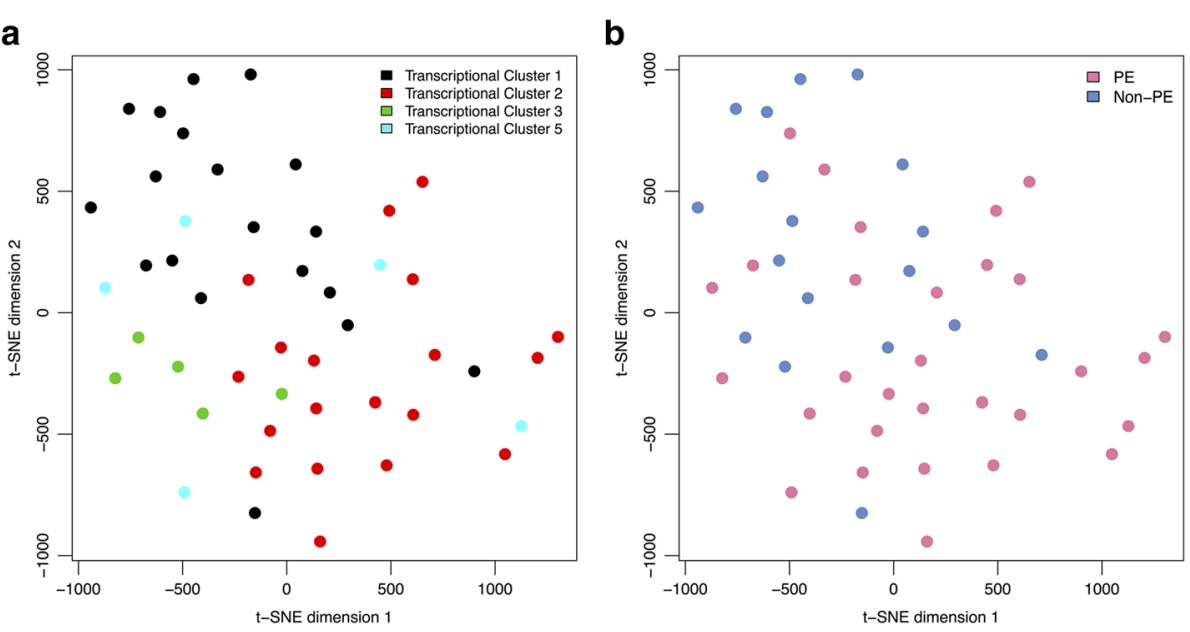

Fig. $1 \mathrm{t}$-Distributed Stochastic Neighbor Embedding (t-SNE) visualization of the methylation data in the 48 placenta samples. a Transcriptional clusters 1 (black, $N=19$ ), 2 (red, $N=19$ ), and 3 (green, $N=5$ ) continued to display molecular similarity to each other based on the DNA methylation data alone, indicating that methylation plays a significant role in the development of these three clusters. Cluster 5 samples (cyan, $N=5$ ), however, were found dispersed across the methylation plot, no longer forming a united group. b In general, preeclamptic (PE) placentas (pink) were found on the bottom half of the t-SNE plot, while the non-PE samples (blue) were predominately observed on the top half. However, the cluster 1 PE patients fully integrated with their co-clustering controls by methylation 
Significance-based modules integrating the transcriptome and epigenome (SMITE)

Differential gene expression between the current subset of transcriptional cluster 2 and 3 samples, compared to cluster 1 placentas, was obtained using the limma library [27], controlling for fetal sex and gestational age. Using the hg19 genome build within the SMITE library [30] in $\mathrm{R}$ 3.3.2, a framework was constructed where each gene was associated with a promoter region $(+/-1500 \mathrm{bp}$ from the TSS) and a gene body region (TSS + $1500 \mathrm{bp}$ to TES). The fetal sex and GA-corrected gene expression and methylation results for clusters 2 and 3 (compared to cluster 1) were then separately integrated into the framework, and the adjusted and combined methylation $p$ values in the promoter and body gene regions were obtained using Stouffer's method, weighted by effect strength. The relationship between expression and methylation was set to "bidirectional" in both gene regions to avoid biasing the results, and genes were scored based on a weighted significance value $(0.4$ for expression, 0.4 for promoter methylation, and 0.2 for body methylation). Gene scores were considered significant at a nominal $p$ value $<0.05$. Functional modules of genes in transcriptional clusters 2 and 3 were then identified based on these gene scores, a Reactome proteinprotein interaction graph [31], and the spin-glass network algorithm. Significant modules (nominal $p<0.05$ and 10-500 genes) were subjected to KEGG pathway enrichment analysis within the SMITE library [30] and terms with a FDR $<0.05$ were held as significant.

\section{Results}

\section{Clinical characteristics and global methylation patterns}

Within this subset of 48 cases, transcriptional cluster 1 patients $(N=19)$ remained the healthiest clinically, with the latest gestational ages at delivery and the highest rates of average-for-gestational-age (AGA) infants (95\%) (Additional file 2: Table S1 and Additional file 3: Table S2). Of these cluster 1 patients, 32\% (6/19) were associated with a diagnosis of $\mathrm{PE}$, though none had co-occurring fetal growth restriction. Cluster $2(N=19)$ and cluster $3(N=5)$ samples demonstrated substantially worse clinical outcomes, with abnormal Doppler ultrasound results, early deliveries (mean $=31$ weeks), and low placental and newborn weights (mean z-scores $<-1.4$ ) (Additional file 2: Table S1 and Additional file 3: Table S2). In cluster 2, 89\% $(17 / 19)$ were diagnosed with PE and exhibited the highest maternal blood pressures (average maximum systolic pressure $=175 \mathrm{mmHg}$ ) and proteinuria levels (average maximum $=+3.5)$. Cluster $3(60 \% \mathrm{PE}(3 / 5))$ was more strongly associated with poor fetal growth, with the largest portion of small-for-gestational-age (SGA) infants (80\%) and NICU transfers after delivery (80\%). Cluster 5 patients $(N=5,80 \% \mathrm{PE})$ continued to display no unique clinical association (Additional file 2: Table S1 and Additional file 3: Table S2). These results are consistent with our previous observations in the full transcriptional clusters [3].

When the global relationships between these 48 patients were visualized using t-SNE of the DNA methylation data only, transcriptional cluster 1,2 , and 3 samples continued to demonstrate molecular similarity to each other (Fig. 1a), indicating that methylation plays an important role in the development of these three clusters. Cluster 5 samples, however, were found dispersed across the methylation plot, no longer forming a united group (Fig. 1a).

\section{Differentially methylated positions between transcriptional clusters}

To identify potential epigenetic markers related to our transcriptional clusters, placentas belonging to clusters 2,3 , and 5 were independently assessed for differentially methylated positions (CpG sites) compared to the healthier cluster 1 . When fetal sex and gestational age were not considered, this revealed a total of 66,837 positions (53,635 hypo- and 13,202 hyper-) with significantly divergent methylation in transcriptional cluster 2 samples compared to cluster 1 (FDR $<0.05$; Additional file 4: Table S3). When fetal sex $(p=0.51$ between clusters 1 and 2) was integrated into the model, this number was reduced to 64,025 , whereas when gestational age $(p<0.01$ between clusters 1 and 2$)$ alone was incorporated, only 8711 significant positions were observed. However, when these two covariates were simultaneously included in the model, the number of significant sites was 8763 (3310 hypo- and 5453 hyper-) (Table 1 and Additional file 4: Table S3). Similar to the reference distribution across the full set of possible probes, the majority of these (fetal sex and gestational age controlled) significant sites were located in a gene body or an intergenic region (all $p>0.05$; Additional file 5: Figure S2a). Conversely, substantially fewer significant positions were annotated to a CpG island $(12 \%$ versus $34 \% ; p<0.01)$ and considerably more to the CpG open sea ( $49 \%$ versus $33 \%$; $p=0.03$ ) than the distribution of the array as a whole (Additional file 5: Figure S2b). Furthermore, $8 \%(735 / 8763)$ of these significant cluster 2 sites were found in a group of at least three significant positions within a span of 1000 base pairs, which were, unsurprisingly, often associated with a $\mathrm{CpG}$ island or shore region $(p<0.01)$ (Table 1 and Additional file 4: Table S3).

In cluster 3 placentas, 13,348 positions were differentially methylated (9084 hypo- and 4264 hyper-) compared to cluster $1($ FDR $<0.05)$ without accounting for fetal sex and GA (Additional file 6: Table S4). The inclusion of fetal sex ( $p=0.12$ between clusters 1 and 3 ) dropped this number to 4343 , while accounting for gestational age $(p=0.02$ between clusters 1 and 3 ) only in the model reduced the significant positions to 1749 . When differences in both these variables were considered, the number of significantly 
Table 1 Top 20 significantly differentially methylated sites in transcriptional cluster 2 placentas $(N=19)$ compared to transcriptional cluster 1 placentas $(N=19)$, corrected for fetal sex and gestational age at delivery

\begin{tabular}{|c|c|c|c|c|c|c|c|c|c|}
\hline Probe & Delta M & Average M & Delta $\beta$ & Average $\beta$ & FDR $q$ value & Gene(s) & ${\text { Location}(s)^{a}}^{a}$ & Enhancer & Group $^{b}$ \\
\hline cg10900537 & 0.41 & 2.75 & 0.03 & 0.87 & 9.93E-04 & FOXN3 & Body-open sea & True & No \\
\hline cg18498598 & 0.35 & 1.80 & 0.03 & 0.78 & $2.15 \mathrm{E}-03$ & CUX1 & Body-open sea & False & No \\
\hline cg11235787 & 0.36 & 1.72 & 0.04 & 0.77 & $2.15 \mathrm{E}-03$ & MIR195 & Body-shelf & False & Yes \\
\hline $\operatorname{cg} 17850498^{c}$ & -0.93 & 1.58 & -0.14 & 0.74 & 2.15E-03 & ECE1 & Body-open sea & True & No \\
\hline cg01938025 & 0.62 & 2.62 & 0.05 & 0.86 & $2.15 E-03$ & SKI & Body-shelf & False & No \\
\hline cg22807822 & 0.61 & 3.49 & 0.03 & 0.92 & 2.15E-03 & KANK2 & Body-shore & False & Yes \\
\hline cg14601621 & 0.54 & 1.79 & 0.08 & 0.77 & $2.15 \mathrm{E}-03$ & C9orf3 & 3'UTR-island & False & Yes \\
\hline cg00483891 & -0.55 & 1.90 & -0.06 & 0.79 & $2.15 \mathrm{E}-03$ & CCDC115 & Body-shore & False & No \\
\hline cg10994126 & -0.63 & -0.23 & -0.13 & 0.46 & $2.92 \mathrm{E}-03$ & PAPPA2 & 1stExon-open sea & False & No \\
\hline cg17107691 & 0.76 & 3.14 & 0.05 & 0.89 & $2.92 \mathrm{E}-03$ & KANK2 & Body-shore & False & Yes \\
\hline cg01412654 & -0.56 & -0.38 & -0.11 & 0.44 & $2.92 \mathrm{E}-03$ & PPARG & TSS1500-shore & False & No \\
\hline cg05359207 & -0.52 & -2.62 & -0.05 & 0.14 & 2.92E-03 & ZNF217 & Body-shore & False & No \\
\hline cg06917772 & -0.35 & -1.33 & -0.06 & 0.29 & $2.92 \mathrm{E}-03$ & MIR3167 & IGR-shore & True & No \\
\hline cg24787238 & 0.34 & 1.29 & 0.04 & 0.71 & $2.92 \mathrm{E}-03$ & MAD1L1 & Body-open sea & True & No \\
\hline cg21564965 & 0.53 & 4.73 & 0.01 & 0.96 & $2.92 \mathrm{E}-03$ & ARHGAP23 & Body-open sea & False & No \\
\hline $\operatorname{cg} 13562353$ & -0.53 & 1.40 & -0.09 & 0.72 & $2.92 \mathrm{E}-03$ & CCL27 & TSS200-shelf & False & No \\
\hline cg26897909 & 0.45 & 1.85 & 0.04 & 0.78 & $2.92 \mathrm{E}-03$ & SRGAP2 & Body-open sea & True & No \\
\hline cg09106999 & -0.44 & -2.01 & -0.05 & 0.20 & $2.92 \mathrm{E}-03$ & $\begin{array}{l}\text { CDK2 } \\
\text { SILV } \\
\text { PMEL }\end{array}$ & $\begin{array}{l}\text { TSS1500-shore } \\
\text { TSS1500-shore } \\
\text { TSS1500-shore }\end{array}$ & False & No \\
\hline cg02006426 & -0.39 & -0.23 & -0.05 & 0.46 & $2.92 \mathrm{E}-03$ & DYSF & IGR-shore & False & No \\
\hline cg19431235 & 0.36 & 1.79 & 0.04 & 0.78 & $2.92 \mathrm{E}-03$ & DIAPH3 & Body-open sea & True & No \\
\hline
\end{tabular}

${ }^{\mathrm{a}}$ TSS transcription start site, IGR intergenic region, UTR untranslated region

b Included in a group of at least three significantly differentially methylated positions within the span of 1000 base pairs

'Also significantly differentially methylated in cluster 3 compared to cluster 1

altered sites in transcriptional cluster 3 further decreased to 340 (164 hypo- and 176 hyper-) (Table 2 and Additional file 6: Table S4). The dispersion of these probes was very similar to the results observed in cluster 2: within the gene-based regions, the (fetal sex and GA corrected) significant sites were randomly distributed (all $p>0.05$; Additional file 7: Figure S3a); however, the number of probes annotated to $\mathrm{CpG}$ islands was lower than random (14\% versus $34 \% ; p<0.01)$ and those located in the CpG open sea was higher (51\% versus $33 \% ; p=0.01$ ) (Additional file 7: Figure S3b). Additionally, $5 \%(16 / 340)$ of these cluster 3 sites were involved in a group of significant positions that were again more likely to be associated with a CpG island region $(p<0.01)$ (Table 2 and Additional file 6: Table S4).

Compared to transcriptional cluster 1 , only four CpG sites were initially identified as differentially methylated in cluster 5 placentas (one hypo- and three hyper-) (FDR $<0.05$; Additional file 8: Table S5), and this number became zero when fetal sex and gestational age were included. This indicates that the gene expression changes that define this cluster are not associated with consistent DNA methylation differences. As such, cluster 5 samples were not investigated further for epigenetic regulation.

\section{Specific functional epigenetic modifications}

In order to identify individual epigenetic changes involved in the transcriptional formation of clusters 2 and 3, significantly differentially methylated sites in these samples compared to cluster 1 were assessed for correlating changes in placental gene expression. Of the 66,837 identified significant positions in transcriptional cluster 2 (before correction for fetal sex and GA), correlative analysis with the expression of all available associated genes revealed only $5 \%$ with a strong linear relationship (FDR $<0.05$; Additional file 9: Table S6). When restricted to the 8763 sites that maintained a significant difference between clusters 1 and 2 after correction for both fetal sex and GA, $9 \%$ of potential DNA methylation values exhibited a significant linear relationship with gene expression (FDR $<0.05$; Table 3 and Additional file 9: Table S6). Positively correlating positions were more frequently found in a $\mathrm{CpG}$ island within a gene body $(p<0.01)$ or in the CpG open sea in a gene body $(p<0.01)$ or intergenic region $(p=0.01)$ (Fig. 2a). Sites with a negative relationship to gene expression were 
Table 2 Top 20 significantly differentially methylated sites in transcriptional cluster 3 placentas $(N=5)$ compared to transcriptional cluster 1 placentas $(N=19)$, corrected for fetal sex and gestational age at delivery

\begin{tabular}{|c|c|c|c|c|c|c|c|c|c|}
\hline Probe & Delta M & Average M & Delta $\beta$ & Average $\beta$ & FDR q value & Gene(s) & Location(s) $^{\mathrm{a}}$ & Enhancer & Group $^{b}$ \\
\hline cg22131172 & 0.79 & -3.59 & 0.04 & 0.08 & $1.58 \mathrm{E}-02$ & $\begin{array}{l}\text { C13orf29 } \\
\text { LINC00346 }\end{array}$ & $\begin{array}{l}\text { TSS1500-open sea } \\
\text { TSS1500-open sea }\end{array}$ & False & Yes \\
\hline cg24079702 & 0.50 & -5.87 & 0.01 & 0.02 & $1.94 \mathrm{E}-02$ & FHL2 & TSS200-island & False & No \\
\hline cg10959820 & 0.75 & -3.98 & 0.03 & 0.06 & $1.94 \mathrm{E}-02$ & RGS12 & IGR-shelf & True & No \\
\hline cg10319331 & 0.63 & -3.84 & 0.02 & 0.07 & 1.94E-02 & TMEM132B & Body-open sea & False & No \\
\hline cg05929019 & -0.80 & -2.79 & -0.05 & 0.13 & $1.94 \mathrm{E}-02$ & LAMC2 & TSS200-open sea & False & No \\
\hline cg22790835 & 0.60 & -2.93 & 0.04 & 0.12 & $1.94 \mathrm{E}-02$ & $\begin{array}{l}\text { C13orf29 } \\
\text { LINC00346 }\end{array}$ & $\begin{array}{l}\text { TSS1500-open sea } \\
\text { TSS1500-open sea }\end{array}$ & False & Yes \\
\hline cg14557185 & 0.78 & -5.55 & 0.01 & 0.02 & $1.94 \mathrm{E}-02$ & WWTR1 & Body-island & False & Yes \\
\hline cg00296578 & 0.74 & -3.60 & 0.04 & 0.08 & $1.94 \mathrm{E}-02$ & CRIM1 & Body-open sea & True & No \\
\hline cg21834463 & -0.47 & 3.31 & -0.03 & 0.91 & $1.94 \mathrm{E}-02$ & SGK1 & Body-open sea & True & No \\
\hline cg12634306 & 1.44 & -4.70 & 0.05 & 0.04 & $1.94 \mathrm{E}-02$ & HEYL & Body-open sea & True & No \\
\hline cg22342100 & 0.83 & -4.06 & 0.03 & 0.06 & $1.94 \mathrm{E}-02$ & KLHL38 & TSS1500-open sea & False & No \\
\hline cg27570256 & -0.67 & 1.50 & -0.10 & 0.74 & $1.94 \mathrm{E}-02$ & LOC100270710 & TSS200-shelf & True & No \\
\hline cg24741430 & 0.66 & -3.09 & 0.05 & 0.11 & $1.94 \mathrm{E}-02$ & SMAD6 & IGR-open sea & True & No \\
\hline cg04082512 & -0.43 & 0.77 & -0.07 & 0.63 & $1.94 \mathrm{E}-02$ & GSE1 & IGR-open sea & True & No \\
\hline cg19458020 & 0.89 & -4.52 & 0.03 & 0.04 & 2.14E-02 & RARA & TSS1500-island & True & No \\
\hline cg25892587 & 0.67 & -3.41 & 0.04 & 0.09 & $2.14 \mathrm{E}-02$ & KLF6 & IGR-open sea & True & No \\
\hline $\mathrm{cg} 07605236^{c}$ & -1.22 & 2.60 & -0.11 & 0.85 & $2.14 \mathrm{E}-02$ & SFXN5 & TSS1500-shore & False & No \\
\hline cg19478410 & -0.61 & 2.34 & -0.07 & 0.83 & $2.16 \mathrm{E}-02$ & SYT13 & IGR-open sea & False & No \\
\hline cg13250752 & 0.57 & -1.98 & 0.06 & 0.20 & 2.16E-02 & PCDH18 & IGR-open sea & True & No \\
\hline cg20669292 & -1.03 & 3.93 & -0.05 & 0.94 & 2.16E-02 & PLEKHH3 & Body-island & True & No \\
\hline
\end{tabular}

${ }^{a} T S S$ transcription start site, IGR intergenic region

b Included in a group of at least three significantly differentially methylated positions within the span of 1000 base pairs

'Also significantly differentially methylated in cluster 2 compared to cluster 1

commonly annotated to a CpG shelf region in a 5'UTR $(p=0.02)$ or a CpG shore region in a $5^{\prime} \mathrm{UTR}(p<0.01)$, TSS1500 ( $p=0.05)$, or TSS200 ( $p=0.01)$ (Fig. 2a). Most significantly correlating positions within the CpG open sea of a gene body or intergenic region were also associated with an enhancer region $(72 \% ; p<0.01$ compared to the other $\mathrm{CpG} /$ gene regions).

In transcriptional cluster 3, the 13,348 significant sites compared to cluster 1 (before correction for fetal sex and GA) showed a strong linear relationship to gene expression only $2 \%$ of the time (Additional file 10: Table S7). This value increased to $8 \%$ when the analysis was restricted to the 340 positions that were significantly differentially methylated between clusters 1 and 3 when simultaneously controlling for fetal sex and GA (Table 4). Only three sites demonstrated a strong positive relationship with expression: one was in the CpG open sea of AFF3's 1st exon ( $p=0.01)$, which was not annotated as an enhancer region, and the other two were in the CpG open sea of the MGST1 gene body $(p=0.11)$ in an enhancer (Fig. 2b). Negatively correlating positions were more frequently associated with an open sea region in a TSS200 $(p=0.02)$, although several were also in gene bodies or the IGR (Fig. 2b).

\section{Integrated functional gene modules}

Lastly, in order to reveal significant functional modules of genes within clusters 2 and 3, their differential gene expression and differential gene promoter and body methylation information, compared to cluster 1 and corrected for fetal sex and GA, were subjected to Significance-based Modules Integrating the Transcriptome and Epigenome (SMITE) analysis [30]. Transcriptional cluster 2 contained 9 significant integrated gene modules $(p<0.05)$, consisting of 18-149 genes each (Fig. 3a and Additional file 11: Figure S4). Modules in this cluster with unique genes $(1,4$, and 6$)$ were associated with TGFbeta signaling, cell adhesion, endocytosis, leukocyte transendothelial migration, and carbohydrate metabolism (Additional file 12: Table S8). Module 3 genes were contained within module 2 , and these were involved in focal adhesion and regulation of the actin cytoskeleton. Modules 5 and 9 were associated with lipid metabolism, while modules 7 and 8 were linked to oxidative phosphorylation and the citrate cycle. The significantly deregulated genes in cluster 2, based on the integrated epigenetic and transcriptional scores, and their module inclusions, are shown in Additional file 13: Table S9. 
Table 3 Top 20 significant gene expression correlations associated with the 8763 significantly differentially methylated sites in transcriptional cluster 2 placentas $(N=19)$ compared to transcriptional cluster 1 placentas $(N=19)$, corrected for fetal sex and gestational age at delivery

\begin{tabular}{|c|c|c|c|c|c|}
\hline Probe & Gene & Location $^{\text {a }}$ & Enhancer & Pearson $r$ & FDR q value \\
\hline cg23677911 & GALNT2 & Body-open sea & True & -0.81 & $3.53 \mathrm{E}-06$ \\
\hline cg26333638 & HEXB & Body-shore & False & -0.81 & $3.53 \mathrm{E}-06$ \\
\hline cg04858987 & SH3BP5 & Body-open sea & True & -0.78 & $1.34 \mathrm{E}-05$ \\
\hline cg13553455 & COL17A1 & TSS1500-open sea & False & -0.78 & $1.34 \mathrm{E}-05$ \\
\hline cg16557964 & TMEM45A & 5'UTR-open sea & True & -0.77 & $1.68 \mathrm{E}-05$ \\
\hline cg19140548 & SH3PXD2A & Body-open sea & True & -0.77 & $2.50 \mathrm{E}-05$ \\
\hline cg15700009 & LDHA & TSS1500-shore & False & -0.76 & $2.70 \mathrm{E}-05$ \\
\hline cg23730027 & FLNB & Body-island & False & -0.76 & 2.93E-05 \\
\hline cg18444702 & SH3BP5 & Body-open sea & True & -0.75 & 4.27E-05 \\
\hline cg17338821 & FLNB & Body-shore & True & -0.75 & 5.14E-05 \\
\hline cg25549791 & GALE & TSS200-shore & False & -0.74 & $5.49 \mathrm{E}-05$ \\
\hline cg14019050 & ABCA1 & TSS1500-island & False & -0.74 & 5.49E-05 \\
\hline cg19512693 & FLT1 & Body-open sea & True & 0.74 & $6.15 \mathrm{E}-05$ \\
\hline cg04704064 & SCARB1 & IGR-island & False & 0.74 & $6.29 \mathrm{E}-05$ \\
\hline cg00411097 & TMEM184A & 1stExon-open sea & True & -0.73 & 7.24E-05 \\
\hline cg11079619 & INHBA & $5^{\prime}$ UTR-shelf & False & -0.73 & $7.32 \mathrm{E}-05$ \\
\hline cg00513984 & SCARB1 & IGR-island & False & 0.73 & $7.32 \mathrm{E}-05$ \\
\hline cg26509870 & PHYHIP & IGR-shelf & False & -0.73 & 8.19E-05 \\
\hline cg06531595 & PDE5A & Body-open sea & True & -0.72 & 9.89E-05 \\
\hline cg18874575 & ZNF559 & 3'UTR-open sea & False & 0.72 & 9.89E-05 \\
\hline
\end{tabular}

${ }^{a}$ TSS transcription start site, IGR intergenic region, UTR untranslated region

In contrast, cluster 3 consisted of 11 significant gene modules $(p<0.05)$, made up of 24-293 genes each (Fig. 3b and Additional file 14: Figure S5). Modules 1, 4, 5,6 , and 7 displayed varying degrees of gene overlap and were all involved in TGF-beta signaling, focal adhesion, and glycosaminoglycan biosynthesis (Additional file 12: Table S8). Modules 2 and 3 were linked to antigen processing/presentation and allograft rejection, while modules 8 and 9 , with $\sim 74 \%$ gene overlap, were associated with cytokine-cytokine receptor interaction and Jak-STAT signaling. Modules in this cluster with unique genes (10 and 11) were involved in purine, amino acid, and biotin metabolism. The significantly deregulated genes in transcriptional cluster 3, based on both gene expression and methylation, and their module inclusions, are also shown in Additional file 13: Table S9.

\section{Discussion}

Our previous work unbiasedly investigating the placental heterogeneity observed in preeclampsia [3] revealed five transcriptional clusters, including four subtypes of PE placentas. However, while gene expression microarrays are an invaluable tool for understanding disease, it is also possible that, in some cases, an alternate level of molecular information is highly involved in the development of the pathology. Combined epigenetic and expression analysis of the same preeclamptic placentas has only ever been performed for a small number of samples [32, 33] or genes [34]. We, therefore, predicted that the integration of matched genome-wide DNA methylation data would further improve our understanding of these placentas, and allow us to investigate both the mechanisms underlying the formation of the transcriptional clusters and the associations between the multimolecular data.

Overall, we found that the relationships between the transcriptional cluster 1-3 samples were still visible within the DNA methylation information, indicating a significant global relationship between the two data types in these samples. Cluster 5 placentas, on the other hand, no longer formed a distinct group by methylation. This is unsurprising given that this data type is known to be fairly robust to copy number abnormalities [35], the driving force behind the molecular formation of this cluster.

Within transcriptional clusters 2 and 3, controlling for fetal sex and gestational age in the identification of differentially methylated sites, compared to the healthier cluster 1 , dramatically reduced the number of significant sites $(66,837$ to 8763 in cluster 2; 13,348 to 340 in cluster 3). However, it predominately corrected the observed imbalance in the 

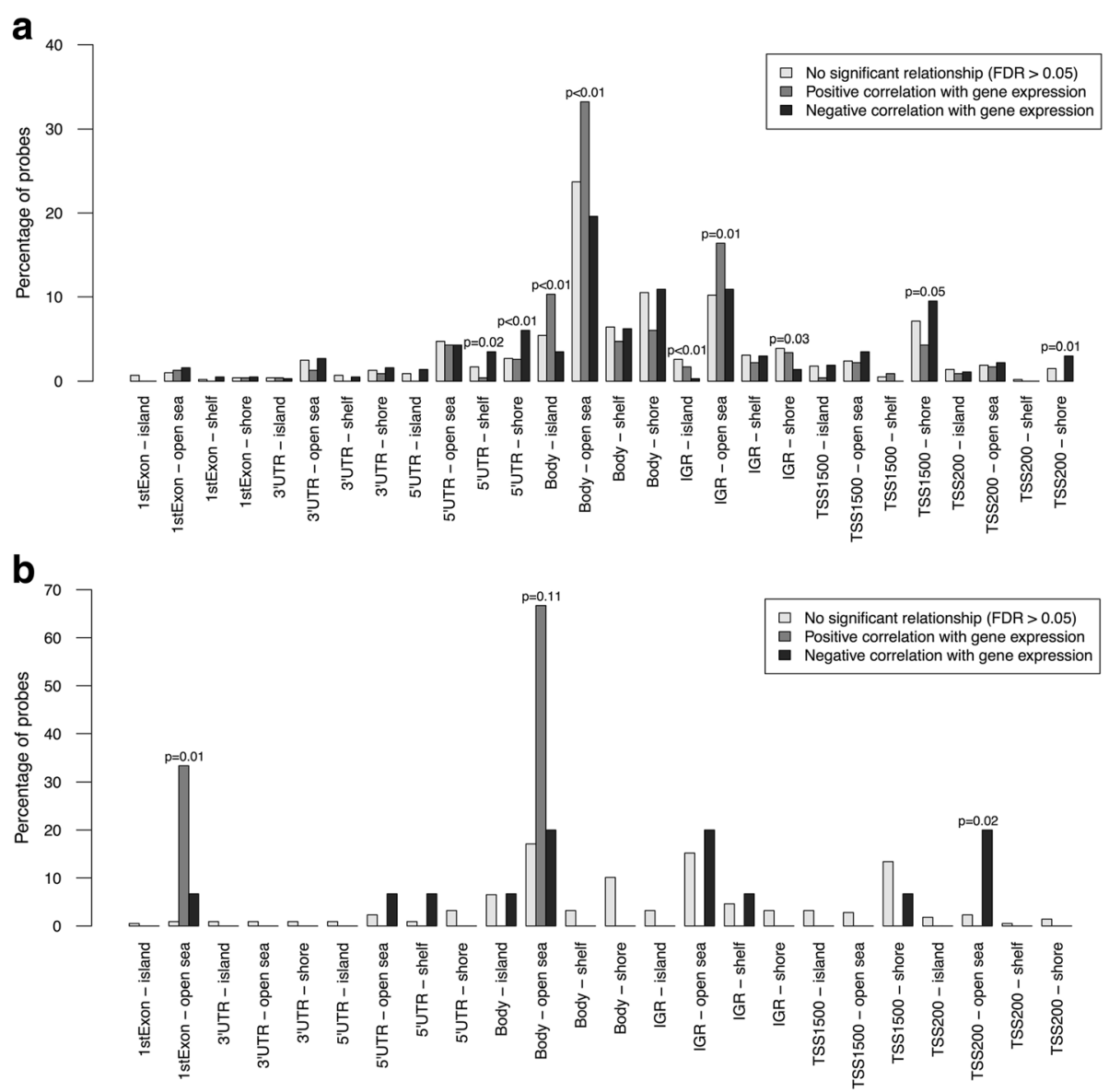

Fig. 2 Distribution of the significantly differentially methylated positions in transcriptional cluster 2 and 3 placentas, compared to cluster 1, in terms of their linear relationships to gene expression. a Within the 8763 (fetal sex and gestational age corrected) significant sites identified in transcriptional cluster 2, positively correlating positions were more frequently found in a CpG island within a gene body or in the CpG open sea in a gene body or intergenic region (IGR). Significant methylation sites with a negative relationship to gene expression were commonly annotated to a CpG shelf region in a 5' untranslated region (UTR) or a CpG shore region in a 5'UTR, transcription start site (TSS)1500, or TSS200. b Within the 340 cluster 3 (fetal sex and gestational age corrected) significant sites, only three demonstrated a strong positive relationship with expression: one was in the CpG open sea of AFF3's 1st exon and the other two were in the CpG open sea of the MGST1 gene body. Negatively correlating positions were more frequently annotated to an open sea region in a TSS200, although several were also in gene bodies or the IGR. Non-significant correlations are shown in light gray, positive correlations are in medium gray, and negative correlations are in dark gray. Nominal $p$ values were obtained from Fisher's exact tests. $P$ values $>0.05$ are not shown

direction of change (80\% hypomethylated to $38 \%$ hypomethylated in cluster 2; $68 \%$ hypomethylated to $48 \%$ hypomethylated in cluster 3$)$. Since both clusters 2 and 3 are significantly younger than cluster $1(p<0.01$ and $p=0.02$, respectively), this fits with the knowledge that placentas become progressively more methylated with time [36], while in cluster 3 , a moderate bias in fetal sex $(p=0.12)$ may have also been involved. Additionally, controlling for fetal sex and GA substantially increased the proportion of significant sites that showed a strong linear relationship with gene expression (5\% to $9 \%$ in cluster $2 ; 2 \%$ to $8 \%$ in cluster 3 ), thereby confirming that a large number of sites in the genome undergo DNA methylation changes in response to differences in these two factors that are independent of epigenetic regulation and gene expression [9, 36, 37].
An additional result of interest was the $\mathrm{CpG}$ distribution of significant positions found in transcriptional clusters 2 and 3. CpG islands are most commonly associated with the regulation of gene expression, especially when located in the gene's promoter region $[8,38]$. We discovered that substantially fewer of the significant sites were mapped into CpG islands than anticipated, based on the reference distribution of all potential CpG sites, although those that were annotated to islands were, unsurprisingly, often found in close proximity to each other. Instead, the majority of significant positions were associated with CpG open sea enhancer regions. This is consistent with a previous report of enrichment of altered DNA methylation at enhancers and low $\mathrm{CpG}$ density regions in early-onset preeclamptic placentas [33]. These open sea enhancer regions, when significantly associated with gene expression, were generally 
Table 4 All significant gene expression correlations associated with the 340 significantly differentially methylated sites in transcriptional cluster 3 placentas $(N=5)$ compared to transcriptional cluster 1 placentas $(N=19)$, corrected for fetal sex and gestational age at delivery

\begin{tabular}{|c|c|c|c|c|c|}
\hline Probe & Gene & Location $^{a}$ & Enhancer & Pearson $r$ & FDR q value \\
\hline cg03983223 & WIPF1 & 1stExon-open sea & FALSE & -0.74 & 4.45E-03 \\
\hline cg05544807 & DNMT3A & Body-island & FALSE & -0.73 & 4.45E-03 \\
\hline $\operatorname{cg} 22462240$ & LGALS3BP & IGR-open sea & FALSE & -0.74 & $4.45 \mathrm{E}-03$ \\
\hline cg18275589 & DAB2 & IGR-shelf & FALSE & -0.72 & $5.02 \mathrm{E}-03$ \\
\hline cg09258479 & PDZK1IP1 & TSS200-open sea & FALSE & -0.69 & $7.40 \mathrm{E}-03$ \\
\hline cg07593977 & CTSB & IGR-open sea & TRUE & -0.69 & 7.40E-03 \\
\hline cg24506086 & TEAD1 & Body-open sea & TRUE & -0.66 & $1.40 \mathrm{E}-02$ \\
\hline cg17850498 & ECE1 & Body-open sea & TRUE & -0.65 & $1.65 \mathrm{E}-02$ \\
\hline cg07349094 & AFF3 & 1stExon-open sea & FALSE & 0.64 & 1.65E-02 \\
\hline cg03821121 & MICAL2 & 5'UTR-open sea & TRUE & -0.65 & $1.65 \mathrm{E}-02$ \\
\hline cg04885072 & MGST1 & Body-open sea & TRUE & 0.63 & $1.94 \mathrm{E}-02$ \\
\hline cg00874480 & MGST1 & Body-open sea & TRUE & 0.62 & 2.45E-02 \\
\hline cg11535839 & FOSL2 & IGR-open sea & FALSE & -0.61 & $2.59 \mathrm{E}-02$ \\
\hline cg05305434 & LSP1 & TSS200-open sea & FALSE & -0.61 & $2.72 \mathrm{E}-02$ \\
\hline cg23170988 & SNCG & Body-open sea & FALSE & -0.60 & 3.17E-02 \\
\hline cg05929019 & LAMC2 & TSS200-open sea & FALSE & -0.59 & $3.75 E-02$ \\
\hline cg15300730 & ZFP36L2 & TSS1500-shore & FALSE & -0.58 & 3.75E-02 \\
\hline cg22234930 & PKM & $5^{\prime}$ UTR-shelf & FALSE & -0.58 & 3.83E-02 \\
\hline
\end{tabular}

${ }^{a}$ TSS transcription start site, IGR intergenic region, UTR untranslated region

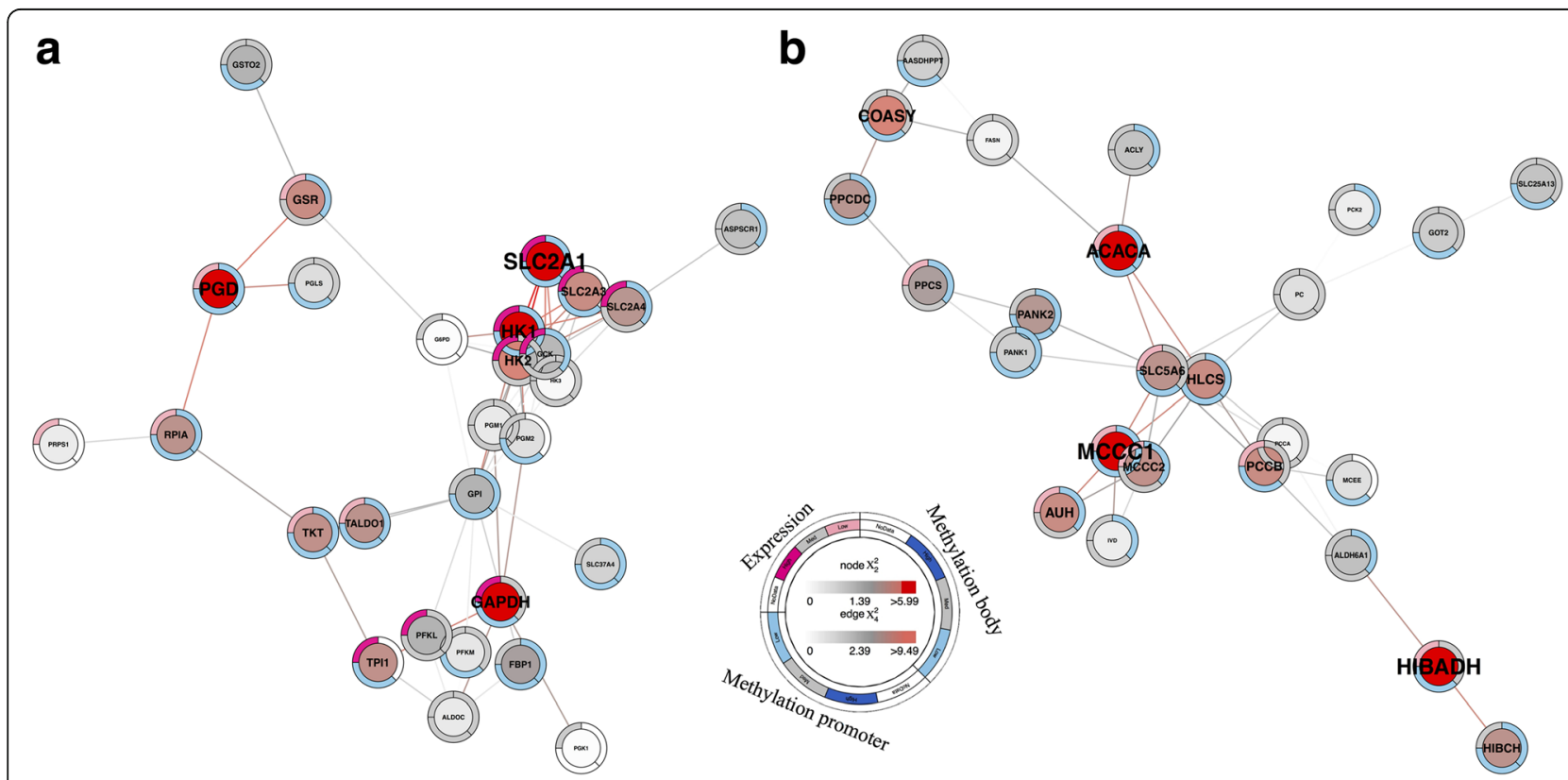

Fig. 3 Example SMITE modules. a Cluster 2 module $6(N=28$ genes; $p=0.02)$ built around SLC2A1 and HK1 genes and involved in carbohydrate metabolism. b Cluster 3 module 11 ( $N=25$ genes; $p=0.04$ ) built around $M C C C 1$ and ACACA genes and involved in amino acid and biotin metabolism. Expression is displayed on the top left edge of each gene circle (upregulated: dark pink; downregulated: light pink; gray: not significant; white: no data), and combined promoter and body methylation are displayed on the bottom left and top right of each circle, respectively (hypermethylated: dark blue; hypomethylated: light blue; gray: not significant; white: no data), compared to cluster 1. The symbol text sizes and center node colors are based on the total gene score (low (gray) to high (red)) and the edge colors are representative of the strength of the associations between the genes (low (gray) to high (red)). The remaining modules are shown in Additional file 11: Figure S4 and Additional file 14: Figure S5 
located in the gene body and exhibited a positive relationship. Sites with a strong negative correlation, on the other hand, were frequently located in the promoter region (TSS200, TSS1500, 5'UTR), as expected, but were annotated to $\mathrm{CpG}$ shore regions, not islands. Relationships between $\mathrm{CpG}$ shores and gene expression are thought to be in response to the binding of transcription factors and changes in the chromatin structure around the promoter $[39,40]$.

While the observed proportions of differentially methylated sites that were associated with corresponding changes in gene expression (2-9\%) are in line with prior studies [9, 32, 41, 42], this indicates that a large number of significant sites in clusters 2 and 3, compared to cluster 1, show no meaningful relationship to gene expression. Some of these DNA methylation alterations could be the consequence of changes in gene expression or function [29, 43, 44], or an adaptive response to maintain stable or rebalanced expression. They could further be remnants of an earlier developmental process, or the result of environmental exposures or treatments, where the transcriptional evidence is no longer measurable [36]. Furthermore, methylation is involved in a range of functions outside of direct transcriptional regulation, such as genome stability [45], splicing [8, 46], and cellular development [47], while gene expression can be regulated by a number of other factors, such as microRNAs [48, 49], transcription factors $[43,50]$, and/or histone modifications $[51,52]$. Therefore, it is expected that these two data types would not fully agree at the individual gene level, although altered methylation sites not associated with changes in gene expression could still provide important information about the overall status and gestational history of these pathological placentas.

When the transcriptome and epigenome data was utilized simultaneously in an integrated analysis, this revealed modifications in TGF-beta signaling, cell adhesion and migration, oxidative phosphorylation, and carbohydrate and lipid metabolism pathways in cluster 2 placentas, confirming that a significant global relationship exists between the two data types. Placental dysfunction encompassing dysregulation of these pathways has been extensively described in the classical paradigm of $\mathrm{PE}$ pathophysiology and fits with our characterization of cluster 2 patients as demonstrating a "canonical" early-onset form of PE [3, 53-59]. Additionally, a number of the top significant methylation and gene expression correlations in this cluster (cg23730027 and FLNB, cg13553455 and COL17A1, cg11079619 and INHBA, cg19140548 and SH3PXD2A, and cg26509870 and PHYHIP) have been previously described in a smaller sample set of early-onset PE placentas [33], thus validating these relationships. We also identified several methylation probes in the gene body of $F L T 1$, one of the most frequently investigated markers of PE, with a strong positive correlation to expression, as well as one associated site in the IGR with a strong negative correlation. These methylation differences could be involved in or attempting to compensate for the pathologically elevated expression of this gene [2, 3], and are significant findings missed by prior studies that have focused only on FLT1 promoter methylation in earlyonset PE [34].

In cluster 3 samples, integrated alterations were identified involving antigen presentation, allograft rejection, cytokinecytokine receptor interaction, Jak-STAT and TGF-beta signaling, glycosaminoglycan biosynthesis, and metabolism. These are also in line with our prior transcriptional results in this "immunological" PE group [3], in which we characterized this cluster of patients as demonstrating evidence of maternal anti-fetal/placental rejection. While not as widely discussed in the literature, the primary involvement of heightened immune activation has been described in several previous studies of PE pathophysiology, along with these other metabolic pathways [60-66]. Interestingly, one of the most significant methylation and expression relationships observed in this cluster involved DNMT3A (one of the DNA methyltransferase enzymes responsible for de novo methylation): a CpG island site (cg05544807) was hypermethylated in the DNMT3A gene body, compared to cluster 1 , and demonstrated a negative relationship to expression. While this likely has global implications for the DNA methylation pattern observed in these cluster 3 placentas, decreased expression of DNMT3A has been specifically implicated in immunological-associated disorders $[67,68]$ and abnormal placentation in preeclampsia [69]. Therefore, this CpG site may serve as a potential target for the epigenetic modulation of pathological gene expression in this PE subtype.

Our study also has some inherent limitations. In our previous gene expression analysis, we utilized a large cohort of over 300 placentas to identify clusters and dysregulated pathways between them. Despite our current study being the largest to integrate methylation and transcriptional information in $\mathrm{PE}$, this analysis involved only 48 placentas. Therefore, it is likely to still be underpowered, thus restricting our discovery of epigenetic changes in these samples to those with large effect sizes. As such, a future direction will be the validation of these findings, and perhaps the identification of new significant sites, in a larger cohort of samples. Additionally, as with all investigations of delivered placentas, it is impossible to determine whether the observed molecular modifications are part of the cause or the consequence of the disease process. Finally, our analysis is based on the assumption that the cell composition is the same across all samples. This is probably not the case, as differences in cell ratios can occur for a range of reasons [4, 42, 70-72], including placental maturation or sampling variability. Therefore, 
some of the epigenetic changes that we are interpreting as being reflective of gene regulation in all cells may instead be due to shifts in cell numbers [29]. However, unfortunately, until individual methylation patterns for all possible placental cell types have been established, this limitation cannot be resolved. This investigation is currently ongoing in our groups.

\section{Conclusions}

Overall, we have improved our understanding of the portion of the divergent gene expression involved in the development of transcriptional clusters 2 and 3 that is associated with changes in DNA methylation, as well as confirmed the lack of true biological cohesion in cluster 5 placentas. Differentially methylated sites in clusters 2 and 3 , compared to the healthier cluster 1 , may have potential as biomarkers of these patient groups for early screening in maternal serum, whereas specific genes and sets of genes exhibiting a strong epigenetic and transcriptional relationship (either linear or integrated) may serve as therapeutic targets to modify or prevent pathological changes in PE placental groups. However, a further increase in sample size and an assessment of additional modes of gene regulation will be required to fully comprehend the mechanisms underlying these subtypes.

\section{Additional files}

\section{Additional file 1: Figure S1. Selected samples for methylation arrays.} (PDF $199 \mathrm{~kb}$ )

Additional file 2: Table S1. Continuous clinical characteristics of the 48 samples across the transcriptional clusters (PDF $72 \mathrm{~kb}$ )

Additional file 3: Table S2. Categorical clinical characteristics of the 48 samples across the transcriptional clusters (PDF $79 \mathrm{~kb}$ )

Additional file 4: Table S3. Significantly differentially methylated sites in transcriptional cluster 2 placentas versus transcriptional cluster 1 placentas. (XLSX $9040 \mathrm{~kb})$

Additional file 5: Figure S2. Distribution of significantly differentially methylated positions in transcriptional cluster 2 (versus transcriptional cluster 1) compared to the full set of possible methylation probes. (PDF $559 \mathrm{~kb})$

Additional file 6: Table S4. Significantly differentially methylated sites in transcriptional cluster 3 placentas versus transcriptional cluster 1 placentas. (XLSX $1669 \mathrm{~kb}$ )

Additional file 7: Figure S3. Distribution of significantly differentially methylated positions in transcriptional cluster 3 (versus transcriptional cluster 1) compared to the full set of possible methylation probes. (PDF $560 \mathrm{~kb})$

Additional file 8: Table S5. Significantly differentially methylated sites in transcriptional cluster 5 placentas versus transcriptional cluster 1 placentas. (XLSX $38 \mathrm{~kb}$ )

Additional file 9: Table S6. Significant gene expression correlations associated with the significantly differentially methylated sites in transcriptional cluster 2 placentas versus transcriptional cluster 1 placentas. (XLSX $259 \mathrm{~kb})$

Additional file 10: Table S7. Significant gene expression correlations associated with the significantly differentially methylated sites in transcriptional cluster 3 placentas versus transcriptional cluster 1 placentas. (XLSX 63 kb)

Additional file 11: Figure S4. Remaining functional SMITE modules identified in cluster 2. (PDF 2447 kb)

Additional file 12: Table S8. Significant KEGG pathways associated with the significant SMITE modules in transcriptional clusters 2 and 3 (XLSX $58 \mathrm{~kb}$ )

Additional file 13: Table S9. Genes with significant integrated gene expression and methylation scores by SMITE analysis in transcriptional clusters 2 and 3. (XLSX $86 \mathrm{~kb}$ )

Additional file 14: Figure S5. Remaining functional SMITE modules identified in cluster 3. (PDF $4125 \mathrm{~kb}$ )

\section{Abbreviations}

aCGH: Array-based comparative genomic hybridization; AGA: Average-forgestational-age; FDR: False discovery rate; GA: Gestational age; IGR: Intergenic region; PE: Preeclampsia; RCWIH: Research Centre for Women's and Infants' Health; SGA: Small-for-gestational-age; SMITE: Significance-based Modules Integrating the Transcriptome and Epigenome; SNP: Single-nucleotide polymorphism; TES: Transcriptional end site; t-SNE: t-distributed stochastic neighbor embedding; TSS: Transcriptional start site; UTR: Untranslated region

\section{Acknowledgements}

We thank the donors and the Research Centre for Women's and Infants' Health (RCWIH) BioBank for the human samples used in this study. We would also like to thank Dr. Maria Peñaherrera for assistance in running the $450 \mathrm{~K}$ arrays.

\section{Funding}

This work was funded by the Canadian Institutes of Health Research (CIHR) grant \#49520 to WPR and the CIHR grant \#128369 to SAB and BJC. KL is supported by an Ontario Graduate Scholarship, SLW is funded by a University of British Columbia Four Year Doctoral Fellowship, WPR receives salary support from the BC Children's Hospital Research Institute, and BJC receives salary support from a Tier 2 Canada Research Chair in Placental Development and Maternal-Fetal Health. The funding bodies had no role in the design of the study, the collection, analysis, and interpretation of the data, and the writing of the manuscript.

\section{Availability of data and materials}

The gene expression microarray data for our full highly annotated sample set $(N=157)$ is available from the Gene Expression Omnibus database under the accession number GSE75010. The matched gene expression and DNA methylation data for the 48 placentas assessed in the current study is available under the accession number GSE98224.

\section{Authors' contributions}

$K L, B J C$, and WPR conceived of the study. SAB and BJC extracted RNA for microarray analysis. KL extracted DNA for methylation analysis. SLW and WPR ran the DNA methylation arrays. $K L$ analyzed the data and drafted the manuscript. BJC, WPR, SLW, and SAB critically revised the manuscript. All authors approved the final manuscript.

\section{Ethics approval and consent to participate}

Ethics approval was granted from the Research Ethics Boards of Mount Sinai Hospital (\#13-0211-E), the University of Toronto (\#29435), and the Ottawa Health Science Network (\#2011623-01H). All women provided written informed consent for the collection of biological specimens and medical information.

\section{Consent for publication}

Not applicable.

\section{Competing interests}

The authors declare that they have no competing interests. 


\section{Publisher's Note}

Springer Nature remains neutral with regard to jurisdictional claims in published maps and institutional affiliations.

\begin{abstract}
Author details
'Department of Physiology, University of Toronto, 1 King's College Circle, Toronto, ON, Canada. ${ }^{2}$ BC Children's Hospital Research Institute, 950 W 28th Ave, Vancouver, BC, Canada. ${ }^{3}$ Department of Medical Genetics, University of British Columbia, C201-4500 Oak St, Vancouver, BC, Canada. Interdisciplinary School of Health Sciences, University of Ottawa, 25 University Private, Ottawa, ON, Canada. ${ }^{5}$ Department of Cellular and Molecular Medicine, University of Ottawa, 451 Smyth Rd, Ottawa, ON, Canada. ${ }^{6}$ Department of Obstetrics and Gynecology, University of Toronto, 23 Edward Street, Toronto, ON, Canada.
\end{abstract}

\section{Received: 1 November 2017 Accepted: 21 February 2018} Published online: 02 March 2018

\section{References}

1. Magee LA, Pels A, Helewa M, Rey E, von Dadelszen P. Diagnosis, evaluation, and management of the hypertensive disorders of pregnancy. Pregnancy Hypertens. 2014;4(2):105-45.

2. Leavey $\mathrm{K}$, Bainbridge SA, Cox BJ. Large scale aggregate microarray analysis reveals three distinct molecular subclasses of human preeclampsia. PLoS One. 2015;10(2):e0116508.

3. Leavey K, Benton SJ, Grynspan D, Kingdom JC, Bainbridge SA, Cox BJ. Unsupervised placental gene expression profiling identifies clinically relevant subclasses of human preeclampsia. Hypertension. 2016;68:137-47.

4. Avila L, Yuen RK, Diego-Alvarez D, Penaherrera MS, Jiang R, Robinson WP. Evaluating DNA methylation and gene expression variability in the human term placenta. Placenta. 2010;31(12):1070-7.

5. Matsui M, Corey DR. Non-coding RNAs as drug targets. Nat Rev Drug Discov. 2017;16(3):167-79.

6. Robertson KD. DNA methylation and human disease. Nat Rev Genet. 2005; 6(8):597-610.

7. Bird AP. DNA methylation versus gene expression. Development. 1984; 83(Supplement):31-40.

8. Jones PA. Functions of DNA methylation: islands, start sites, gene bodies and beyond. Nat Rev Genet. 2012;13(7):484-92.

9. Lim YC, Li J, Ni Y, Liang Q, Zhang J, Yeo GS, et al. A complex association between DNA methylation and gene expression in human placenta at first and third trimesters. PLoS One. 2017;12(7):e0181155.

10. Schultz MD, He Y, Whitaker JW, Hariharan M, Mukamel EA, Leung D, et al. Human body epigenome maps reveal noncanonical DNA methylation variation. Nature. 2015;523(7559):212-6.

11. Yuen RK, Chen B, Blair JD, Robinson WP, Nelson DM. Hypoxia alters the epigenetic profile in cultured human placental trophoblasts. Epigenetics. 2013;8(2):192-202.

12. Van Neste L, Herman JG, Otto G, Bigley JW, Epstein JI, Van Criekinge W. The epigenetic promise for prostate cancer diagnosis. Prostate. 2012;72(11):1248-61.

13. Heyn $\mathrm{H}$, Esteller M. DNA methylation profiling in the clinic: applications and challenges. Nat Rev Genet. 2012;13(10):679-92.

14. Yang X, Lay F, Han H, Jones PA. Targeting DNA methylation for epigenetic therapy. Trends Pharmacol Sci. 2010;31(11):536-46.

15. Issa JP. DNA methylation as a therapeutic target in cancer. Clin Cancer Res. 2007;13(6):1634-7.

16. Rechache NS, Wang Y, Stevenson HS, Killian JK, Edelman DC, Merino M, et al. DNA methylation profiling identifies global methylation differences and markers of adrenocortical tumors. J Clin Endocrinol Metab. 2012;97(6): E1004-13.

17. Wilson SL, Leavey K, Cox B, Robinson WP. Mining DNA methylation alterations towards a classification of placental pathologies. Hum Mol Genet. 2018;27(1):135-46

18. Morris TJ, Butcher LM, Feber A, Teschendorff AE, Chakravarthy AR, Wojdacz TK, et al. ChAMP: 450k chip analysis methylation pipeline. Bioinformatics. 2013;30(3):428-30

19. Nordlund J, Bäcklin CL, Wahlberg P, Busche S, Berglund EC, Eloranta ML, et al. Genome-wide signatures of differential DNA methylation in pediatric acute lymphoblastic leukemia. Genome Biol. 2013;14(9):r105.

20. Price EM, Cotton AM, Lam LL, Farré P, Emberly E, Brown CJ, et al. Additional annotation enhances potential for biologically-relevant analysis of the
Illumina Infinium HumanMethylation450 BeadChip array. Epigenetics Chromatin. 2013;6(1):4.

21. Fortin JP, Labbe A, Lemire M, Zanke BW, Hudson TJ, Fertig EJ, et al. Functional normalization of 450k methylation array data improves replication in large cancer studies. Genome Biol. 2014;15(11):503.

22. Leek JT, Johnson WE, Parker HS, Jaffe AE, Storey JD. The sva package for removing batch effects and other unwanted variation in high-throughput experiments. Bioinformatics. 2012;28(6):882-3.

23. Du P, Zhang X, Huang CC, Jafari N, Kibbe WA, Hou L, et al. Comparison of Beta-value and M-value methods for quantifying methylation levels by microarray analysis. BMC Bioinformatics. 2010;11(1):587.

24. Zhuang J, Widschwendter M, Teschendorff AE. A comparison of feature selection and classification methods in DNA methylation studies using the Illumina Infinium platform. BMC Bioinformatics. 2012;13(1):59.

25. Gautier L, Cope L, Bolstad BM, Irizarry RA. affy — analysis of Affymetrix GeneChip data at the probe level. Bioinformatics. 2004;20(3):307-15.

26. Maaten LV, Hinton G. Visualizing data using t-SNE. J Mach Learn Res. 2008;9: 2579-605.

27. Ritchie ME, Phipson B, Wu D, Hu Y, Law CW, Shi W, et al. Limma powers differential expression analyses for RNA-sequencing and microarray studies. Nucleic Acids Res. 2015:43(7):e47.

28. Singmann P, Shem-Tov D, Wahl S, Grallert H, Fiorito G, Shin SY, et al. Characterization of whole-genome autosomal differences of DNA methylation between men and women. Epigenetics Chromatin. 2015;8(1):43.

29. Wilson SL, Robinson WP. Utility of DNA methylation to assess placental health. Placenta. 2017; https://doi.org/10.1016/j.placenta.2017.12.013.

30. Wijetunga NA, Johnston AD, Maekawa R, Delahaye F, Ulahannan N, Kim K, et al. SMITE: an R/Bioconductor package that identifies network modules by integrating genomic and epigenomic information. BMC Bioinformatics. 2017;18(1):41.

31. Fabregat A, Sidiropoulos K, Garapati P, Gillespie M, Hausmann K, Haw R, et al. The reactome pathway knowledgebase. Nucleic Acids Res. 2015;44(D1): D481-7.

32. Xuan J, Jing Z, Yuanfang Z, Xiaoju H, Pei L, Guiyin J, et al. Comprehensive analysis of DNA methylation and gene expression of placental tissue in preeclampsia patients. Hypertens Pregnancy. 2016;35(1):129-38.

33. Blair JD, Yuen RK, Lim BK, McFadden DE, von Dadelszen P, Robinson WP. Widespread DNA hypomethylation at gene enhancer regions in placentas associated with early-onset pre-eclampsia. Mol Hum Reprod. 2013;19(10): 697-708.

34. Sundrani DP, Reddy US, Joshi AA, Mehendale SS, Chavan-Gautam PM, Hardikar AA, et al. Differential placental methylation and expression of VEGF, FLT-1 and KDR genes in human term and preterm preeclampsia. Clin Epigenetics. 2013;5(1):6.

35. Blair JD, Langlois S, McFadden DE, Robinson WP. Overlapping DNA methylation profile between placentas with trisomy 16 and early-onset preeclampsia. Placenta. 2014;35(3):216-22.

36. Novakovic B, Yuen RK, Gordon L, Penaherrera MS, Sharkey A, Moffett A, et al. Evidence for widespread changes in promoter methylation profile in human placenta in response to increasing gestational age and environmental/stochastic factors. BMC Genomics. 2011;12(1):529.

37. Martin E, Smeester L, Bommarito PA, Grace MR, Boggess K, Kuban K, et al. Sexual epigenetic dimorphism in the human placenta: implications for susceptibility during the prenatal period. Epigenomics. 2017;9(3):267-78.

38. Esteller M. CpG island hypermethylation and tumor suppressor genes: a booming present, a brighter future. Oncogene. 2002;21(35):5427.

39. Irizarry RA, Ladd-Acosta C, Wen B, Wu Z, Montano C, Onyango P, et al. The human colon cancer methylome shows similar hypo-and hypermethylation at conserved tissue-specific CpG island shores. Nat Genet. 2009:41(2):178-86.

40. Robinson WP, Price EM. The human placental methylome. Cold Spring Harb Perspect Med. 2015;5(5):a023044.

41. Edgar R, Tan PP, Portales-Casamar E, Pavlidis P. Meta-analysis of human methylomes reveals stably methylated sequences surrounding CPG islands associated with high gene expression. Epigenetics Chromatin. 2014;7(1):28.

42. Lam LL, Emberly E, Fraser HB, Neumann SM, Chen E, Miller GE, et al. Factors underlying variable DNA methylation in a human community cohort. Proc Natl Acad Sci. 2012;109(Suppl 2):17253-60.

43. Moarii M, Boeva V, Vert JP, Reyal F. Changes in correlation between promoter methylation and gene expression in cancer. BMC Genomics. 2015;16(1):873. 
44. Timp W, Feinberg AP. Cancer as a dysregulated epigenome allowing cellular growth advantage at the expense of the host. Nat Rev Cancer. 2013; 13(7):497-510

45. Putiri EL, Robertson KD. Epigenetic mechanisms and genome stability. Clin Epigenetics. 2011;2(2):299.

46. Luco RF, Allo M, Schor IE, Kornblihtt AR, Misteli T. Epigenetics in alternative pre-mRNA splicing. Cell. 2011;144(1):16-26.

47. Smith ZD, Meissner A. DNA methylation: roles in mammalian development. Nat Rev Genet. 2013;14(3):204-20.

48. Bartel DP. MicroRNAs: genomics, biogenesis, mechanism, and function. Cell. 2004;116(2):281-97.

49. Chen K, Rajewsky N. The evolution of gene regulation by transcription factors and microRNAs. Nat Rev Genet. 2007;8(2):93-103.

50. Karin M. Too many transcription factors: positive and negative interactions. New Biol. 1990;2(2):126-31.

51. Berger SL. Histone modifications in transcriptional regulation. Curr Opin Genet Dev. 2002;12(2):142-8.

52. Heintzman ND, Hon GC, Hawkins RD, Kheradpour P, Stark A, Harp LF, et al. Histone modifications at human enhancers reflect global cell-type-specific gene expression. Nature. 2009;459(7243):108-12.

53. Caniggia I, Grisaru-Gravnosky S, Kuliszewsky M, Post M, Lye SJ. Inhibition of TGF- $\beta 3$ restores the invasive capability of extravillous trophoblasts in preeclamptic pregnancies. J Clin Investig. 1999;103(12):1641.

54. Zhou X, Li Q, Xu J, Zhang X, Zhang H, Xiang Y, et al. The aberrantly expressed miR-193b-3p contributes to preeclampsia through regulating transforming growth factor- $\beta$ signaling. Sci Rep. 2016;6:19910.

55. Kang JH, Song H, Yoon JA, Park DY, Kim SH, Lee KJ, et al. Preeclampsia leads to dysregulation of various signaling pathways in placenta. J Hypertens. 2011;29(5):928-36.

56. Bloxam DL, Bullen BE, Walters BN, Lao T. Placental glycolysis and energy metabolism in preeclampsia. Am J Obstet Gynecol. 1987;157(1):97-101.

57. Korkes HA, Sass N, Moron AF, Câmara NO, Bonetti T, Cerdeira AS, et al. Lipidomic assessment of plasma and placenta of women with early-onset preeclampsia. PLoS One. 2014;9(10):e110747.

58. Riquelme G, Vallejos C, De Gregorio N, Morales B, Godoy V, Berrios M, et al. Lipid rafts and cytoskeletal proteins in placental microvilli membranes from preeclamptic and IUGR pregnancies. J Membr Biol. 2011;241(3):127.

59. Kim MS, Yu JH, Lee MY, Kim AL, Jo MH, Kim M, et al. Differential expression of extracellular matrix and adhesion molecules in fetal-origin amniotic epithelial cells of Preeclamptic pregnancy. PLoS One. 2016;11(5):e0156038.

60. Laresgoiti-Servitje E. A leading role for the immune system in the pathophysiology of preeclampsia. J Leukoc Biol. 2013;94(2):247-57.

61. Conrad KP, Benyo DF. Placental cytokines and the pathogenesis of preeclampsia. Am J Reprod Immunol. 1997:37(3):240-9.

62. Kim CJ, Romero R, Chaemsaithong P, Kim JS. Chronic inflammation of the placenta: definition, classification, pathogenesis, and clinical significance. Am J Obstet Gynecol. 2015;213(4):S53-69.

63. Wilczyński JR. Immunological analogy between allograft rejection, recurrent abortion and pre-eclampsia-the same basic mechanism? Hum Immunol. 2006;67(7):492-511.

64. Gleicher N. Why much of the pathophysiology of preeclampsia-eclampsia must be of an autoimmune nature. Am J Obstet Gynecol. 2007;196(1):5-e1-7.

65. Heyer-Chauhan N, Ovbude IJ, Hills AA, Sullivan MH, Hills FA. Placental syndecan-1 and sulphated glycosaminoglycans are decreased in preeclampsia. J Perinat Med. 2014;42(3):329-38.

66. Pérez-Sepúlveda A, España-Perrot PP, Fernández BX, Ahumada V, Bustos V, Arraztoa JA, et al. Levels of key enzymes of methionine-homocysteine metabolism in preeclampsia. Biomed Res Int. 2013;2013:731962.

67. Nawrocki MJ, Majewski D, Puszczewicz M, Jagodziński PP. Decreased mRNA expression levels of DNA methyltransferases type 1 and $3 A$ in systemic lupus erythematosus. Rheumatol Int. 2017:37(5):775-83.

68. Liu Y, Chen Y, Richardson B. Decreased DNA methyltransferase levels contribute to abnormal gene expression in "senescent" CD4+ CD28- T cells. Clin Immunol. 2009;132(2):257-65.

69. Jia Y, Li T, Huang X, Xu X, Zhou X, Jia L, et al. Dysregulated DNA methyltransferase $3 \mathrm{~A}$ upregulates IGFBP5 to suppress trophoblast cell migration and invasion in preeclampsia. Hypertension. 2017;69(2):356-66.

70. Adalsteinsson BT, Gudnason $H$, Aspelund T, Harris TB, Launer LJ, Eiriksdottir $\mathrm{G}$, et al. Heterogeneity in white blood cells has potential to confound DNA methylation measurements. PLoS One. 2012;7(10):e46705.
71. Reinius LE, Acevedo N, Joerink M, Pershagen G, Dahlén SE, Greco D, et al. Differential DNA methylation in purified human blood cells: implications for cell lineage and studies on disease susceptibility. PLoS One. 2012;7(7): e41361.

72. Varley KE, Gertz J, Bowling KM, Parker SL, Reddy TE, Pauli-Behn F, et al. Dynamic DNA methylation across diverse human cell lines and tissues. Genome Res. 2013;23(3):555-67.

\section{Submit your next manuscript to BioMed Central and we will help you at every step:}

- We accept pre-submission inquiries

- Our selector tool helps you to find the most relevant journal

- We provide round the clock customer support

- Convenient online submission

- Thorough peer review

- Inclusion in PubMed and all major indexing services

- Maximum visibility for your research

Submit your manuscript at www.biomedcentral.com/submit
) Biomed Central 